\title{
Physically informed car engine sound synthesis for virtual and augmented environments
}

Conference Paper · March 2015

DOI: 10.1109/SIVE.2015.7361287

CITATIONS

0
READS

354

4 authors:

\section{Stefano Baldan}

University of Milan

8 PUBLICATIONS 5 CITATIONS

SEE PROFILE

Stefano Delle Monache

Università luav di Venezia

25 PUBLICATIONS 125 CITATIONS

SEE PROFILE
Helene Lachambre

Institut de Recherche en Informatique de To...

20 PUBLICATIONS 37 CITATIONS

SEE PROFILE

Patrick Boussard

GENESIS Acoustics,France, Aix-en-Provence

26 PUBLICATIONS 48 CITATIONS

SEE PROFILE

Some of the authors of this publication are also working on these related projects:

Project SkAT-VG - Sketching Audio Technologies using Vocalizations and Gestures View project

Project SkAT-VG - Sketching Audio Technologies using Vocalizations and Gestures View project 


\section{Physically informed car engine sound synthesis for virtual and augmented environments}

\author{
Stefano Baldan* \\ IUAV University of Venice, Italy
}

\author{
Hélène Lachambre ${ }^{\dagger}$ \\ Genesis, France
}

\author{
Stefano Delle Monache $\ddagger$ \\ IUAV University of Venice, Italy
}

\author{
Patrick Boussard $\$$ \\ Genesis, France
}

\begin{abstract}
The richness of crossmodal feedback in car driving makes it an engaging, complex, yet "natural" activity. Audition plays an important role, as the engine sound, perceived in the cabin, conveys relevant cues about the vehicle motion. In this paper, we introduce a procedural and physically informed model for synthetic combustion engine sound, as an effective, flexible and computationally efficient alternative to sample-based and analysis/resynthesis approaches. The sound model, currently being developed as Max/MSP external, has been integrated in GeneCars, a driving simulator environment for industrial sound design, and SkAT Studio, a demonstration framework for the rapid creation of audio processing workflows.
\end{abstract}

Index Terms: H.5.5 [Information Interfaces and Presentation]: Sound and Music Computing-Modeling;

\section{INTRODUCTION}

Car driving is a complex, yet "natural" activity, which requires a tight control loop. Drivers exploit the available sensory cues (visual, auditory, haptic and proprioceptive) to effectively perceive the vehicle motion and behavior, avoid obstacles and potential collisions, signal their presence to pedestrians and other drivers. What may sound so obvious nowadays, is actually the result of over a century of investigation in the perceptual and cognitive aspects of the driver's behavior $[14,7]$. In this context, driving simulators and virtual environments have been developed and exploited to support driver's training [17, 23], to assess and evaluate the driving performance under diverse conditions, such as imminent collisions [19], lack of sleep [1] or alcohol assumption [27], to improve the overall quality of vehicles and the productive processes in car manufacturing, and to provide realistic platforms for entertainment applications.

Despite the importance of vision in the driving experience, it has been shown how the perceived sound affects the driving task, and contributes to shape convincing and effective simulations [10]. In particular, being strictly linked to the vehicle motion, the noise resulting from the engine rotations provides the driver with a reference cue in keeping the speed steady. Without the need of constantly monitoring the speedometer, the visual load of the driver can remain focused on the road [9].

In this respect, car manufacturers are investing a lot of energy in providing hybrid and electric vehicles (HEV), which are inherently silent, with a voice of their own ${ }^{1}$. Indeed, although a quieter vehicle might appear more comfortable, the lack of a distinguishable and

\footnotetext{
*e-mail: stefanobaldan@iuav.it

†e-mail: helene.lachambre@genesis.fr

$\ddagger$ e-mail: sdellemonache@iuav.it

$\S$ e-mail: patrick.boussard@genesis.fr
}

\footnotetext{
${ }^{1}$ http: //www youtube. com/watch?v=HoEDLvQZg5I: The Audi e-Tron sound. Audi is developing the synthetic sound signature for its future electric cars.
}

informative motion noise deprives the driver of important information. Moreover, silent vehicles may present safety issues for bikers and pedestrians, indeed the sound produced at low speed is almost unnoticeable to the ear, especially if one considers the average level of noise pollution in our everyday environments [21,20]. A careful sound design for both the interior and exterior should please the ears, as well as being able to prevent dangerous situations.

For this purpose, the most recent generation of cars are provided with embedded solutions for Active Sound Design (ASD) [3, 5], which exploit the car audio system to reduce the original mechanical sound, and modify or even replace it with virtual engine sounds, in order to improve both the brand and driving experience [24]. For example, the R-SOUND optional of the new Renault Clio 4 allows to alter the original engine sound and disguise it with a motorbike or a sports car character.

In this paper, we introduce a procedural and physically-informed model for the synthesis of combustion engine sounds. This approach is an effective, flexible and computationally efficient alternative to sample-based and analysis/resynthesis techniques, and is suitable for applications in virtual environments. The sound model, currently being developed as Max/MSP external, has been integrated in GeneCars, a driving simulator environment, and SkAT Studio, a demonstration framework for the rapid creation of audio processing workflows. The progressive availability of these systems not only will provide designers with new solutions, but also with sketching and prototyping tools to be exploited in the conceptual stage of the design process.

The paper is organized as follows: Section 2 provides an overview on combustion engine sound synthesis, highlighting some strengths and weaknesses of the diverse approaches; section 3 describes in detail our sound model, its design and implementation; in Section 4, we briefly describe two use cases of integration in virtual environments for sound design purposes; finally, in the last section we draw our conclusions.

\section{Related WORKS}

Combustion engine sound simulators can be roughly split in two main groupings: Sample-based and procedural. In sample-based approaches, wavetable techniques are exploited to manipulate existing recordings of real engine sounds. Sound samples are typically looped, cross-faded and pitch-shifted according to control information such as revolutions per minute (RPM), throttle load and gear [18]. This approach is largely used in video games [8] and also in some of the most advanced and high-fidelity driving simulators [16], it is relatively simple to implement and provides very realistic results. However, the main drawbacks are that good simulations require huge banks of sound samples, and that the sound design space is heavily constrained by the timbre of the recorded samples.

In procedural audio approaches, sound is generated starting from a computed description of the engine sound [11]. Analysis/resynthesis techniques exploit the harmonic, stationary nature of engine sounds and rely on their spectrogram as a description of the acoustic event. Manipulating engine harmonics in amplitude and frequency is a flexible way to explore the sound design possibilities within the boundaries of a well-defined space $[2,4]$. 
An advanced real-time sound synthesis tool is GeneCars, software environment developed by the French industrial sound design company Genesis [13]. As shown in figure 1, the synthetic powertrain sound is derived by the analysis of real engine sound samples and integrated with other components such as engine starter, wipers, aerodynamic noise, tire rolling and screeching, traffic noise and so on. Spatial audio processing enhances the virtual sonic experience of driving.

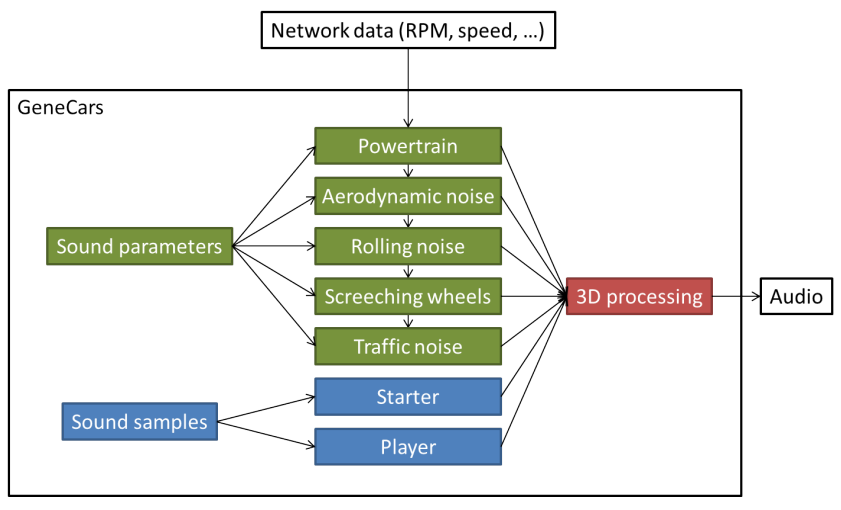

Figure 1: GeneCars synthesizer overview.

The powertrain sound (engine partials and noise) is generated through additive synthesis, wherein Bark-scale partials are conditioned in frequency and amplitude as a function of the engine's RPM, and pedal force load.

A complementary approach to procedural audio exploits the physics involved in mechanical interactions to generate the sound $[26,15]$. Physically informed modeling of synthetic engine sounds is based on physical events and dimensions, allows the exploration of wide sound design spaces with a single model, and potentially provides a more intuitive control on the sound synthesis process. However, most of the work done in this direction is either covered by patents [6] or largely undocumented. An exception is represented by Andy Farnell's engine sound model [12], a well documented example, shown in figure 2. The basic assumption underlying Farnell's physical model is that the salient acoustic contribution in a four-stroke engine is represented by the exhaust gas, expelled at high pressures by the upward motion of the pistons through the exhaust valves and resonating inside the exhaust system. On the contrary, the contribution of fuel ignition is less significant, since the actual explosions occur in perfectly sealed combustion chambers, made of a very thick and dense material. Since the exhaust valves open only during every other positive pressure cycle of the piston, the contribution of each cylinder in Farnell's model is represented by the positive half cycle of a cosine wave, once every two full cycles. The signal is then fed into a delay chain with feedback, split in four quadrants, which simulates the resonances of the pipes in the exhaust system.

This very coarse approximation of the four-stroke cycle, however, results in a timbre which is poor in higher order harmonics. A mixture of pseudo-physical solutions, FM operators and waveshaping techniques are used to overcome this limitation, although making the overall control of the model quite difficult to master, often with unnatural and hardly predictable acoustic results.

\section{Physically informed SyNTHEsis OF MOTOR SOUNDS}

Farnell's engine sound model represented the starting point of our work, towards a framework which would take into account:

1. A more detailed description of the cylinders, their configuration and functioning;

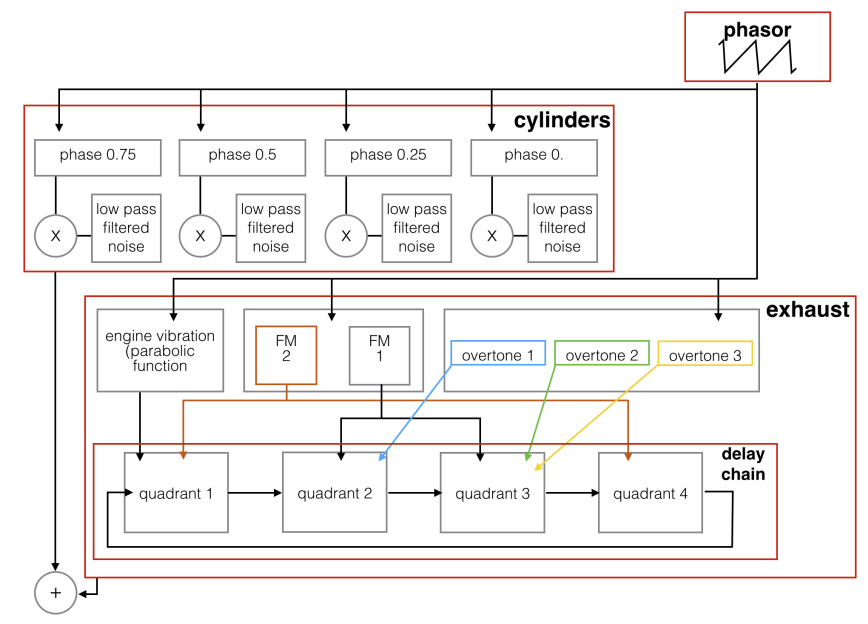

Figure 2: Block diagram of Andy Farnell's engine sound synthesis model.

2. A more accurate representation of resonating tubes;

3. A more realistic layout of the interactions occurring between the engine components.

\subsection{The four-stroke cycle}

Our first step towards the improvement of the starting model was to replace the signal-based operators with physically-consistent processing. For this purpose, we complemented the functioning of the exhaust valves with a more detailed representation of the cylinder's mechanics. Therefore we focused on the actual acoustic contributions of fuel ignition, intake valves operation and piston motion, as salient features of the four-stroke engine sound, which are described as follows:

Exhaust valve: The motion is represented by the positive half of a sine wave during the last quarter of the engine operation cycle:

$$
e(x)= \begin{cases}-\sin (4 \pi x), & \text { if } 0.75<x<1 \\ 0 & \text { otherwise }\end{cases}
$$

Intake valve: It is represented similarly, except that the function is shifted so that the movement happens during the first quarter of the cycle, as it occurs in an actual four-stroke engine:

$$
i(x)= \begin{cases}\sin (4 \pi x), & \text { if } 0<x<0.25 \\ 0 & \text { otherwise }\end{cases}
$$

Piston motion: Being the piston attached to a rotating crankshaft, the resulting motion is harmonic and can be also represented by a sine wave. At the beginning of the first stroke the piston is at its maximum height, with a backward and forward motion twice at every cycle of the engine:

$$
p(x)=\cos (4 \pi x)
$$

Fuel ignition: It causes a sudden increase in pressure into the combustion chamber. As the combustion is fast, though not perfectly instantaneous, this event is represented as the positive half of a sine wave, shifted at the beginning of the expansion phase and rescaled by a parameter $t$, which represents 
the time (relative to the full engine cycle) needed by the fuel to explode:

$$
s(x)= \begin{cases}\sin (2 \pi(x t+0.5)), & \text { if } 0<x<t \\ 0 & \text { otherwise }\end{cases}
$$

The speed at which the engine operates depends on a phasor which sets the variable $x$. Each ramp of the phasor corresponds to two full revolutions of the crankshaft, according to the principles of operation of a four-stroke engine. The engine speed is normally expressed in revolutions per minute, while frequency in audio signals is represented by cycles per second, therefore the phasor's frequency is set according to the following conversion:

$$
f=\frac{\mathrm{RPM}}{120}
$$

Figure 3 shows the shape and alignment of the four functions (intake, exhaust, fuel ignition, piston motion), which represent the moving components of a four-stroke engine.
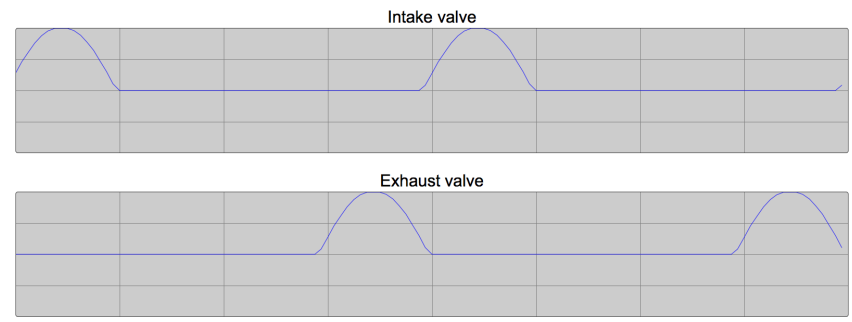

Fuel ignition

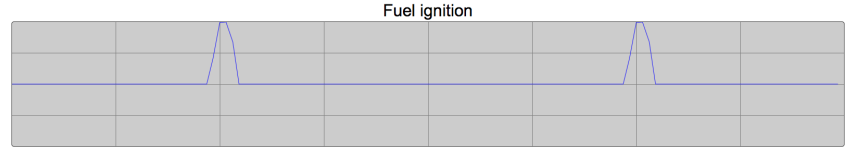

Piston movement

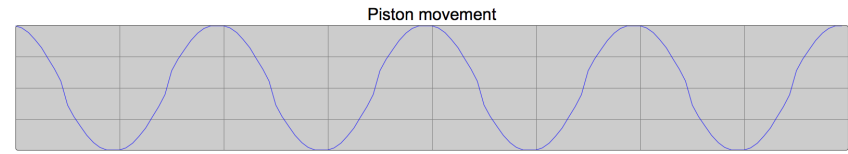

Figure 3: Functions simulating the moving components of a fourstroke engine.

\subsection{Modeling the resonances}

From the acoustic point of view, an internal combustion engine can be described as a set of interconnected pipes, whose resonant modes are excited by the aerodynamic interactions occurring during the engine operation. In particular, the exhaust system is an essential element, which not only affects the engine's performance, but also contributes to shape the "voice" and identity of a vehicle. In our model, tube resonances are simulated through a set of digital waveguides [25].

In its most basic form, a digital waveguide is composed by two delay lines with adjustable gain, wherein the output of each line is fed into the input of the other. The working principle of a digital waveguide approximates the interactions occurring inside a real pipe: The acoustic wave enters from one of the two ends, takes some time to travel across the whole section and bounces back, with a degree of energy dissipation during the process. As shown in figure 4 , a positive gain factor preserves the phase of the signal, thus representing the closed end, whereas a negative gain factor inverts the phase of the signal and simulates the open end. The delayed wave creates a series of constructive and destructive interferences with the original signal, which amplify the resonant modes of the tube, while attenuating anything in between.

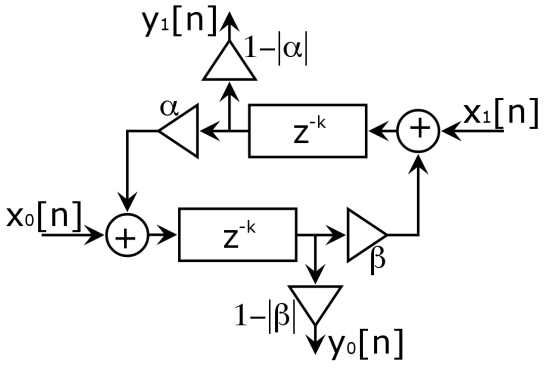

Figure 4: Block diagram of the digital waveguide implemented in the current version of the engine model.

Although simpler approaches to resonance simulations have been investigated, such as modal synthesis or comb filters, they didn't provide the desired sound quality. Moreover, digital waveguides can be connected together in a bidirectional way, thus creating complex mutual acoustic interactions between resonating objects. This kind of connection can be realized both in series and in parallel. The serial connection between two waveguides is done by feeding the forward output of the first waveguide into the forward input of the second, and by feeding the reverse output of the second into the reverse input of the first. To obtain parallel connections, it is sufficient to sum all the parallel outputs into a single receiving input, or to equally divide a single output by all the parallel receiving inputs.

\subsection{Redesigning the model}

In our implementation, the relevant components of the engine are treated as resonant cavities and represented by mutually interacting digital waveguides. Inputs, delays and feedback coefficients of each element are then determined and modulated by the four-stroke cycle operation functions. The aim is to provide a sufficiently realistic and harmonically rich engine sound without any post processing, and to obtain a meaningful and intuitive control parameters. Figure 5 represents the overall structure of the proposed model. In the following paragraphs, each functional block will be described in further detail.

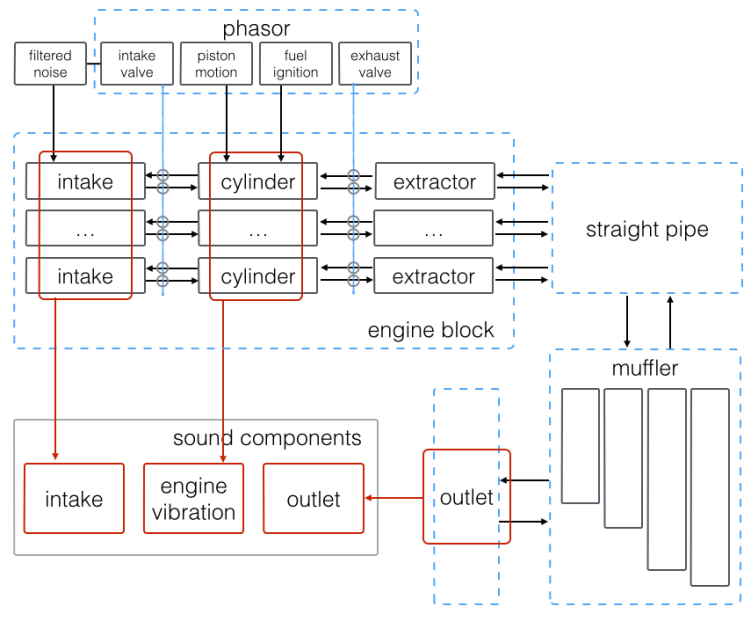

Figure 5: Blocks diagram of the proposed model.

Sound generation starts in the waveguides representing the cylinders. Piston motion and fuel ignition, as described in section 3.1, 
affect the pressure level in the combustion chamber and are thus fed as inputs to the forward delay line of the corresponding waveguide. Ignitions are modulated in amplitude by the throttle load, to simulate stronger explosions when more fuel is given to the engine. Piston motion also affects the volume of the combustion chamber and therefore the delay time of the corresponding waveguide. Intake and exhaust valves are then used to modulate the feedback coefficients: When the valve is open, most of the signal is sent through the corresponding output instead of being recirculated, whereas when the valve is closed most of the energy is fed back to the delay lines.

Most engines are made of more than one cylinder. Normally, the operation cycles of the cylinders are equally shifted in phase in order to uniformly distribute power to the crankshaft. Nevertheless, engine revolutions are rarely smooth and precise, due to the intrinsically chaotic combustion process and to the unavoidable imperfections in the design and construction of an engine. To take account of these facts, the phase shifting of the cylinders can be made slightly asymmetric along the full cycle of the engine. This results in a sound with an oscillatory character at low regimes and a "growling" voice at higher rates, typical of engines of large size, such as in trucks, buses, muscle cars or chopper motorbikes.

Each cylinder is connected to an intake collector and to an exhaust collector. They are modeled as waveguides of fixed length, with fixed feedback on the free end ( -0.5 for intakes, 0.1 for extractors) and variable feedback on the valve end, consistently modulated by the corresponding intake or exhaust valve together with the connected cylinder. Intakes are fed with a secondary source of input, namely lowpass-filtered white noise, modulated in amplitude by the intake valve function. This signal simulates the turbulence in the air and fuel mixture caused by the aspiration inside the combustion chamber. The summed output of the free ends of the intake collectors is the first output of the model, and represents the sound at the listening point located under the hood of the vehicle. A second output is obtained by lowpass-filtering the sum of pistons motions and fuel ignitions, and represents the sound produced by the vibrations of the engine block. These vibrations are mainly transmitted through the chassis of the vehicle, and are mainly heard in the cabin.

The exhaust system is composed of three main parts: a straight pipe, a set of muffler elements and a final outlet. Extractors are connected in parallel to the straight pipe, which is in turn connected in parallel to the muffler elements. Finally, the muffler elements are connected in parallel to the outlet. The output coming from the free end of the outlet represents the third output of the model, namely the sound at the listening point located at the rear of the vehicle.

The muffler plays a fundamental role in shaping the exhaust sound. In a real vehicle, it is composed by a set of tubes designed to partially reflect acoustic waves and create interferences in order to cancel certain frequencies and reduce exhaust noise. In general, tube lengths and cavity areas are specifically tuned for a particular engine, in order to attenuate a precise set of frequencies. Figure 6 represents the basic components of an exhaust muffler.

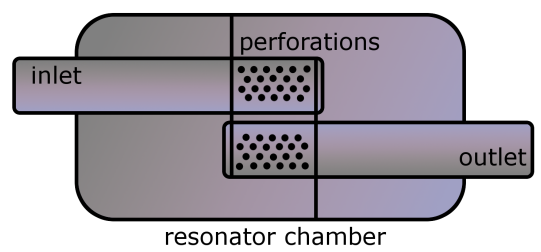

Figure 6: Simplified structure of an exhaust muffler.

In our model, the action of the exhaust muffler is rendered by means of four independent, partially reflecting waveguides. Zero reflection provides almost no silencing, while a feedback factor of 1 results in a perfectly silent muffler. Delay lines are set so that every frequency has a peak in the frequency response of at most one element, in order to maximize destructive interferences.

A simulation of exhaust backfiring is also provided. Backfiring is a phenomenon which occurs especially in the high performance engines of racing or muscle cars. In some cases, the fuel mixture does not burn completely in the cylinders and self ignites later in the hotter parts of the exhaust system. Backfiring is modeled as a Poisson process. When the engine is revving down, that is when the RPM are decreasing, at every full cycle a probability factor is tested against a random value. If the probability factor is greater than the random value the engine backfires, and an impulsive signal, similar to the fuel ignition function used inside the cylinders, is added to the input of the exhaust outlet. At every backfire the probability factor is multiplied by itself, yielding an exponential decrease to make backfire events less likely to happen over time.

We compared the sampled sound of an idling car with our synthetic sound, resulting from a virtual engine configuration matching the kind and size of the recorded one. Although they sound very similar, there is a slight difference in brightness and in the presence of engine block vibrations. Figure 7 shows the spectrograms of the two sounds, which share a similar pattern although the synthesized sound has a lower energy concentration in the medium-high frequencies.

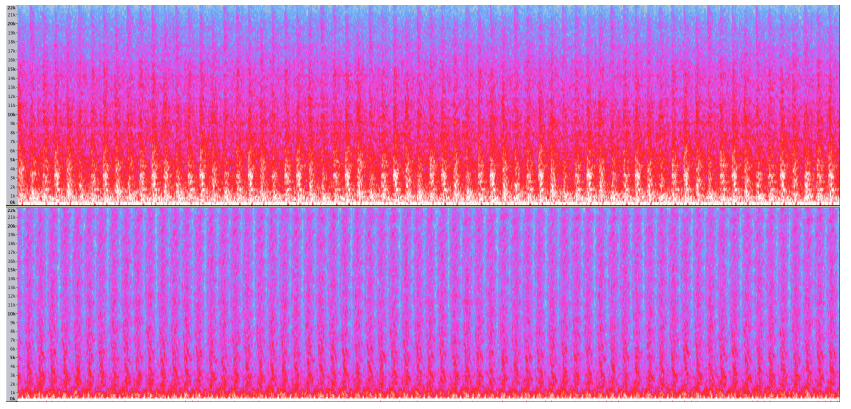

Figure 7: Up: spectrogram of the idling engine (at about 900 RPM) of a medium-sized family car, recorded with a Zoom $\mathrm{H} 2$ recorder at about one meter over the engine with the hood open. Down: spectrogram of the synthetic sound, generated with a configuration matching the characteristics of the recorded car.

\section{A SOUND MODEL FOR VIRTUAL ENVIRONMENTS}

This sound model has been designed to maximise the flexibility of procedural audio approaches in virtual environments. The current release of the engine sound model has been implemented as Max/MSP external and it is controllable according to the following parameters and dimensions:

Cylinders: Number (1 to 12), volume in cubic centimeters, compression (ratio between chamber volume with piston at the bottom of its stroke and with piston at the top of its stroke), ignition time (width of the explosion impulse, with 0 being instantaneous and 1 taking the full engine operation cycle), cycle asymmetry (with 0 being perfectly symmetric and 1 making cylinders overlap, determines the amount of engine "growl"), engine block gain;

Intakes: Average length in meters, gain;

Extractors: Average length in meters;

Exhaust: Length of the main pipe in meters; 
Muffler: Average length in meters, action (with 0 being very loud and 1 being completely silent);

Outlet: Length in meters, gain;

RPM: Revolutions per minute of the crankshaft;

Throttle: Ranging from 0 to 1 , with 0 corresponding to idling and 1 to full throttle.

Vehicle sound design in entertainment applications, such as racing video games or driving simulators, can rely on a single model only to populate the traffic and driving scenes, without the need of actually collecting sound recordings. RPM and throttle load, typically the engine operation descriptors, can be directly used as control data, unlike sample-based synthesis approaches which require further processing and the appropriate playback rates. Moreover, the modular configuration of the outputs (intakes, engine block and exhaust) allows to dynamically render the driving scene from different listening points (i.e. interior, exterior, front, rear). Sound designers are potentially provided with a versatile and intuitive model to start with, when approaching the conceptual stage of the design process. In addition, the proposed sound model can be integrated in future Active Sound Design applications, and allow the driver to customize the flavor of the sound, by simply altering few characteristics of the virtual engine.

In the following subsections, we will briefly describe two integration examples of our engine model in sonic virtual environment applications, namely GeneCars, a pre-existing driving simulator, and SkAT Studio, a demonstration framework for the rapid creation of audio processing workflows, currently being developed in the context of multidisciplinary research on sketching practices for sound design.

\subsection{GeneCars}

GeneCars is a sonic virtual environment for driving simulation. The system, partially developed in Max/MSP, is composed of two main parts:

1. The engine sound synthesizer, complemented with all the relevant noises typically present in a driving scene, e.g. starter, road, motion of the wheels (see section 2);

2. GenePilot, the interface used to interactively drive the synthesis by means of steering wheel and pedals. The pilot is essentially a dynamic model that provides the synthesizer with the basic control data such as engine RPM, pedal load and car speed based on the gear number and the throttle position.

Figure 8 shows the typical listening environment of the driving simulation system, and the GUI of the software environment. Sound effects such as traffic, rain, wind, wet road, wipers and so forth can be added at user's choice, to enrich the sound simulation.

Given the modularity of GeneCars (synthesizer + pilot), we replaced the existing powertrain model with ours. The integration is facilitated by shared programming environment (Max/MSP) and high level control parameters (RPM and normalized throttle load). Informal evaluations and listening tests stressed the effectiveness of sound model, with a convincing degree of realism, low computational cost, and no audible delay. On the other side, we plan to improve the integration of our model in pilots by completing the presets with other relevant control information (e.g. engine inertia, gear ratio, minimum and maximum RPM), to preserve an overall veridical behavior of the simulated engines.

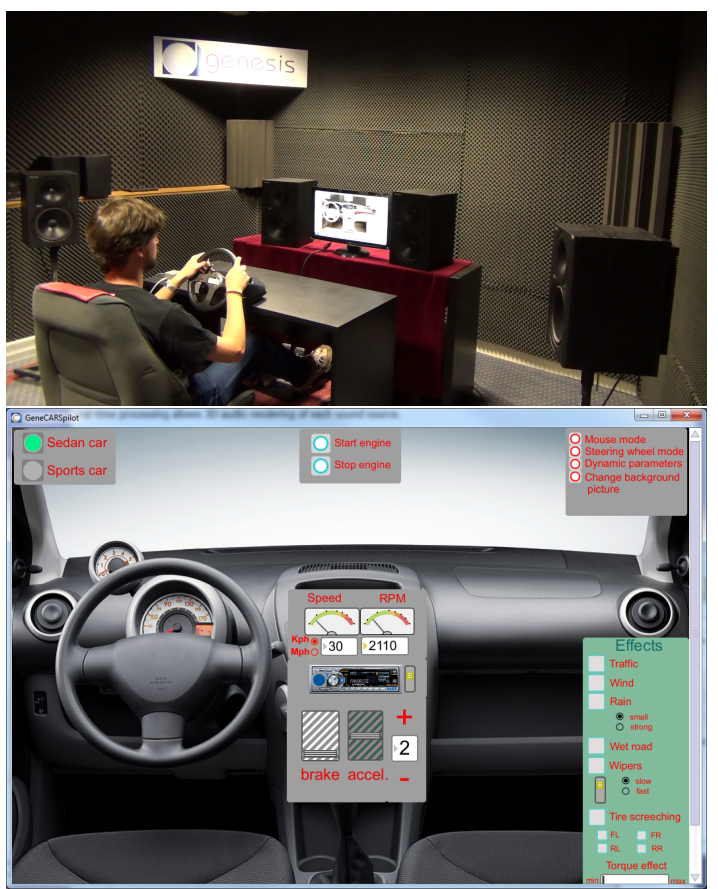

Figure 8: Up: user testing the sound in the listening environment. Down: GeneCars GUI.

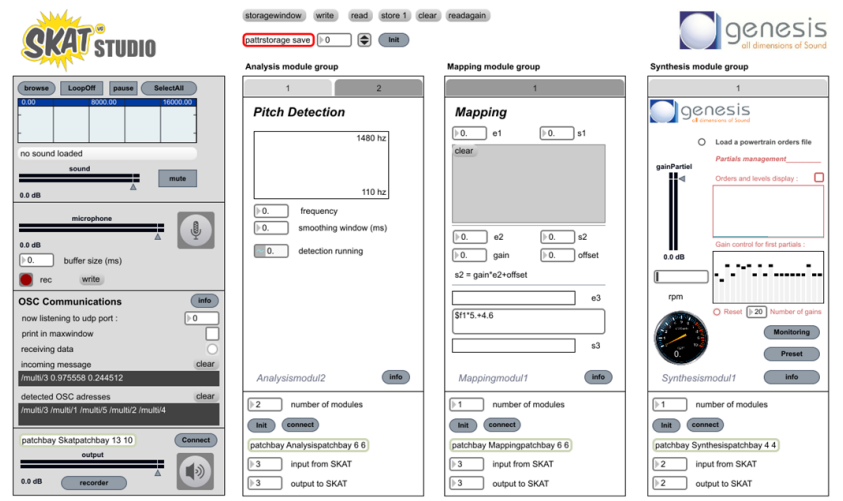

Figure 9: Audio processing workflow in SkAT Studio.

\subsection{SkAT Studio}

SkAT Studio is a modular framework for the quick creation of audio processing workflows in Max/MSP. Its ongoing development is framed in the field of multidisciplinary research on computational support to sound design practices, especially regarding the early and conceptual stages of the design process. In this context, a promising paradigm for sketching purposes is represented by the use of vocalizations and gestures to drive synthetic sound models [22]. Indeed, voice-converted sound models can be further refined, easily communicated, and potentially exchanged among designers and stakeholders.

As shown in figure 9, the demonstration tool is composed of four main sections which describe the processing chain: vocal input, live or recordings (1); analysis, wherein audio features extraction modules can be added $(2,3)$; mapping, wherein extracted audio features activity is conditioned into meaningful control parameters (4); models for sound synthesis (5); output.

Engine sound design is a use case scenario emerged from a set 
of interviews conducted with several professional sound designers, and currently under exploration through the use of SkAT Studio. A possible application, devised by some interviewees, is to exploit the voice to actually control the engine synthesis in time-consuming tasks, such as syncing car sounds in animated movies. For this purpose, we realized a demonstration which exploits the vocal activity (pitch detection) as means to control the RPM of the engine model. This tool will be exploited in forthcoming workshops on motor sound design, wherein we expect to collect valuable insights for the improvement of the sound model.

\section{Conclusions}

Despite the large use of procedural approaches in computer graphics (e.g., 3D modeling, virtual reality, interactive visualization), there is still a certain reluctance in exploiting similar approaches in sound creation. Physics-based synthesis has been traditionally investigated and developed in the context of music computing and composition, although it is finding its way in non-musical fields too. Certainly, the design of virtual and augmented environments may benefit from the introduction of solutions that are inherently flexible and economical. In this spirit, we presented a synthetic model for combustion engine sounds, based on the computed, yet simplified description of the mechanics underlying the functioning of the actual four-stroke engines. As far as we know, this is one of the few documented works that make use of a physically informed approach to engine sound synthesis. The sound model has been implemented as Max/MSP external and we plan to make it available soon for download, in order to receive feedback from users. We plan to improve the computational efficiency and the sound quality of the model, with particular attention to the backfiring algorithm and to the engine block vibrations. We plan to refine the two demonstrations towards applicative results, in virtual applications such as simulators and computational tools for sound design, and augmented environments, such as the ASD systems embedded in real vehicles.

\section{ACKNOWLEDGEMENTS}

The authors are pursuing this research as part of the project SkAT-VG and acknowledge the financial support of the Future and Emerging Technologies (FET) programme within the Seventh Framework Programme for Research of the European Commission, under FET-Open grant number: 618067.

\section{REFERENCES}

[1] T. Åkerstedt, B. Peters, A. Anund, and G. Kecklund. Impaired alertness and performance driving home from the night shift: a driving simulator study. Journal of sleep research, 14(1):17-20, 2005.

[2] S. A. Amman and M. Das. An efficient technique for modeling and synthesis of automotive engine sounds. IEEE Transactions on Industrial Electronics, 48(1):225-234, 2001.

[3] P. Audrain. Active sound design. Technical report, SAE Technical Paper, 2011.

[4] Y. Ban, H. Banno, K. Takeda, and F. Itakura. Synthesis of car noise based on a composition of engine noise and friction noise. In ICASSP'02, pages 2105-2108, 2002.

[5] P. Boussard, S. Molla, and F. Orange. Comprehensive process for car engine sound design: from signal processing to an audio system integrated in the vehicle. In Internoise, pages 7092-7099, 2012.

[6] K. Cascone, D. T. Petkevich, G. P. Scandalis, T. S. Stilson, K. F. Taylor, and S. A. Van Duyne. Apparatus and methods for synthesis of internal combustion engine vehicle sounds, Oct. 25 2005. US Patent $6,959,094$.

[7] C. Castro. Human factors of visual and cognitive performance in driving. CRC Press, 2008.

[8] K. Collins. Game sound: an introduction to the history, theory, and practice of video game music and sound design. Mit Press, 2008.
[9] S. Denjean, V. Roussarie, R. Kronland-Martinet, S. Ystad, J.-L. Velay, et al. How does interior car noise alter driver's perception of motion? multisensory integration in speed perception. Acoustics 2012 Nantes, 2012.

[10] S. Denjean, J.-L. Velay, R. Kronland-Martinet, V. Roussarie, J.-F. Sciabica, S. Ystad, et al. Are electric and hybrid vehicles too quiet for drivers? In Internoise 2013, 2013.

[11] A. Farnell. Behaviour, structure and causality in procedural audio. In M. Grimshaw, editor, Game sound technology and player interaction concepts and developments, pages 313-329. Information Science Reference, New York, NY, USA, 2010.

[12] A. Farnell. Designing Sound. MIT Press, Cambridge, MA, 2010.

[13] B. Gauduin and P. Boussard. High fidelity sound rendering for car driving simulators. In Driving Simulation Conference DSC, pages 283-294, 2009.

[14] J. J. Gibson and L. E. Crooks. A theoretical field-analysis of automobile-driving. The American journal of psychology, pages 453471, 1938.

[15] G. Guyader. Modlisations temporelles simplifies des phnomnes physiques l'origine du bruit moteur: ddies au portage temps rel et aux applications de design sonore (in french). $\mathrm{PhD}$ thesis, INSA, Villeurbanne, 2003.

[16] D. A. Heitbrink and S. Cable. Design of a driving simulation sound engine. In Driving Simulation Conference, North America 2007 (DSCNA 2007), 2007.

[17] K. Ivancic IV and B. Hesketh. Learning from errors in a driving simulation: Effects on driving skill and self-confidence. Ergonomics, 43(12): 1966-1984, 2000.

[18] J. Jagla, J. Maillard, and N. Martin. Sample-based engine noise synthesis using an enhanced pitch-synchronous overlap-and-add method. The Journal of the Acoustical Society of America, 132(5):3098-3108, 2012.

[19] J. D. Lee, D. V. McGehee, T. L. Brown, and M. L. Reyes. Collision warning timing, driver distraction, and driver response to imminent rear-end collisions in a high-fidelity driving simulator. Human Factors: The Journal of the Human Factors and Ergonomics Society, 44(2):314-334, 2002.

[20] L. Maffei, M. Masullo, M. Di Gabriele, and F. Sorrentino. Preliminary studies on the relation between the audio-visual cues' perception and the approaching speed of electric vehicles. The Journal of the Acoustical Society of America, 134(5):3978-3978, 2013.

[21] N. Misdariis, A. Cera, E. Levallois, and C. Locqueteau. Do electric cars have to make noise? an emblematic opportunity for designing sounds and soundscapes. In Acoustics 2012, 2012.

[22] D. Rocchesso, G. Lemaitre, P. Susini, S. Ternström, and P. Boussard. Sketching sound with voice and gesture. interactions, 22(1):38-41, 2015.

[23] D. L. Roenker, G. M. Cissell, K. K. Ball, V. G. Wadley, and J. D. Edwards. Speed-of-processing and driving simulator training result in improved driving performance. Human Factors: The Journal of the Human Factors and Ergonomics Society, 45(2):218-233, 2003.

[24] R. Schirmacher. Active design of automotive engine sound. In Audio Engineering Society Convention 112, Apr 2002.

[25] J. O. Smith. Physical modeling using digital waveguides. Computer music journal, pages 74-91, 1992.

[26] J. O. Smith. Physical audio signal processing. Linear Predictive, 2010.

[27] J. M. Weiler, J. R. Bloomfield, G. G. Woodworth, A. R. Grant, T. A. Layton, T. L. Brown, D. R. McKenzie, T. W. Baker, and G. S. Watson. Effects of fexofenadine, diphenhydramine, and alcohol on driving performancea randomized, placebo-controlled trial in the iowa driving simulator. Annals of Internal Medicine, 132(5):354-363, 2000. 